\title{
Commentary
}

\section{Evolutionary conservative phenomena in eukaryotic cells and evo- lutionary cell biology}

\author{
LI JINGYAN
}

Kunming Institute of Zoology, Academia Sinica, Kunming 650107, China

\section{The significance of evolutionary viewpoint in cell research and the evolutionary conserva- tive phenomena at cellular and subcellular levels}

Evolutionary theory is one of the fundamental principles of modern biology, and therefore, the evolutionary viewpoint is one of the basic viewpoints in the research of biological problems.

In living organisms, all the organic structures at various levels, from macromolecules (nucleic acids, proteins and polysaccharides), the complexes of macromolecules (nucleosomes, ribsomes, membranes), organelles, cells, tissues, organs, systems, up to organisms, are all the results of evolution. The mode and the mechanism of any bio-activity of an organic structure are also the achievements of evolution. They all have their respective evolutionary histories, which are of importance in furthering our comprehension.

When one kind of human cells was compared with the corresponding analogous cells of lower vertebrates or even with those of invertebrates, it was often found to be quite similar in general morphology. The flagella of various flagellates, the cilia of different groups of ciliates, the flagella of sperms of various animals, mosses and ferns and the cilia of ciliated epithelia of different groups of metazoa, all possess similar basic architectures, although they are diversified in details. All the nuclei of different phyla of eukaryotes, from the nuclei of diplomonads (e.g. Giardia)to those of human nerve cells, are similar in morphology and physiology, with only very rare exceptions (e. g. dinoflagellate nucleus). Highly conservative phenomena can also be observed in other kinds of organelles. Similar conservative phenomena were also found in the basic processes of molecular biology. For example, the DNA replication process within human cell nucleus is very similar to the same process in yeast nucleus or in any other protist nucleus.

Clearly some architectures of cellular and subcellular organic structures and the metabolic mechanisms do not change during the prolonged historical stage. How can we harmonize these phenomena with the evolution theory and explain them from evolutionary viewpoint?

At first, it has to be pointed out that highly conserved phenomenon, in any circumstances, does not mean the organic structures or processes concerned have not had their own development in the early stages of their histories. For example, the highly conservative phenomenon of cell nucleus appeared only after the typical eukaryotic nucleus had already formed. Nuclei must have their own origin and evolutionary history during the 
Evolutionary conservative phenomena in eukaryotic cells

early genesis of eukaryotes. It is likely that even DNA replication process had its own evolutionary history. Therefore, the existence of these highly conservative phenomena by no means precludes the significance of the evolutionary theory, and these phenomena in eukaryotic cells do not refute the importance of evolutionary viewpoint in studying the biology of eukaryotic cells.

\section{The proposal of evolutionary cell biology}

One of the major missions of cell biologists is to probe into the evolutionary history of eukaryotic cells, including the origin and diversfication of varius organelles, complexes of macromolecules, and cellular and subcellular activities. This is also an important function of evolutionary biology, because the emergence of eukaryotic cells, i.e. the genesis of primitive unicellular eukaryotes, was one of the epoch-making events in the evolution of organisms.

At the borderline area of cell biology and evolutionary biology, the evolutionary cell biology lays great stress on the necessity of evolutionary viewpoint in studying the architectures of various organic structures within cells and in investigating the modes and mechanisms of various cellular and subcellular activities.

Take mitosis as an example. The details of mitotic process presents itself not only as the basis in molecular biology, but also a keystone in evolutionary history. There are a series of different types of mitosis in lower eukaryotes, representing different branches of the evolutionary tree of mitosis. The mechanisms of chromosome separation and segregation in the different types of mitosis are widely divergent. For example, the typical dinoflagellate extranuclear mitosis $[1,2]$ is quite different from the typical mitosis in multicellular eukaryotes. The special intranuclear mitosis of special dinoflagellate Oxyrrhis marina [3] is still far different from the typical dinoflagellate mitosis as well as from that in ordinary eukaryotes, while somewhat nearer to the typical intranuclear mitosis in many protists. There are remarkable differences between the molecular bases of different types of mitosis. All these dissimilarities are also the result of evolution. Ensuring which type of mitosis is more primitive and elucidating the mechanism and the molecular biological basis of the ancestral mitosis evolved into the advanced types, will undoubtly help us to comprehend in depth the mechanisms of mitosis.

Evolutionary cell biology provides a new domain for further enriching and developing the present-day molecular cell biology. Its contents and the characteristics in the methods of research have been presented elsewhere [4, 5]. The main purpose of the present paper is to probe the essence and the engenderment of the highly conservative phenomena in eukaryotic cells.

\section{The emergence of evolutionary conservative phenomena}

All the biological structures and processess have evolved along the evolutionary lines to become more and more suitable and effective for carrying out their respective functions. However, the suitableness and effectiveness as result of evolution are always only relative, and never perfect. Thus, theoretically, evolution ought to be ever progressive and infinite. But in reality, it is not always the case. The architectures of cytological structures and the mechanisms of living activities as described above, are generally highly 
conserved.

There are some factors which prevent the architectures and the mechanisms from evolving progressively.

\section{The mitigation of the functional contradiction and the genesis of evolutionary conser- vation}

( 1 ). The functional contradiction and its intensification

Besides those features which adapt biological structures or processes to their respective functions, there are also inappropriate aspects, which are very obvious in various evolutionarily initial structures or processes. For example, the appendages of the ancestor of terrestrial vertebrates, Osteolepis (Crossptergii) were inadaptable for the locomotor function on land, and the primitive limbs of ancestral amphibian Ichthyostega (Labyrinthodontia) still could barely undertake this function. The suitable and unsuitable aspects bring about the functional contradiction of each organic structure or process.

The functional contradictions could be greatly intensified in a series of cases. For example, if the functional requirements of an organic structure was greatly enhanced due to the alteration of environment (e. g. , from aquatic to terrestrial environment), or in the case that the organic structure undertook a new function or that its less important function had become a principal one (e. g. , swimming replaced flying as the principal function of the wings of the ancestor of penguin). Such intensified functional contradictions will determine the focal points of evolution.

A mutation that tends to mitigate the intensified functional contradiction may bring a selective advantage to the individual, and, therefore, will stand the best chance to be favoured in natural selection. This means that beneficial mutations will be preserved with an unusually high probability. Consequently, the mutations which reduce the intensity of functional contradiction, are inevitably bound to be accumulated in the genome.

The primitive eukaryote lacking mitochondria serves as another example. Here, the contradiction between the practical capacity of the energy-supplying system (which could hardly carry out oxidative phosphorylation) and the great energy requirement was very intense. The low energy supply kept the organisms at very low metabolic level. At this evolutionary stage, the focal point of evolution was to mitigate this intensified functional contradiction. The mutations which raised energy supply would effect a great selective advantage to the individual and would be preserved with an exceedingly high probability and certainly be accumulated. This was the promoting force to establish endosymbiotic bacteria as mitochondria. During this evolution the endosymbiotic bacteria were selected not in accordance with the interests of themselves, but with the benefit of the host, as these bacteria had already lost the capacity to live an independent life due to the loss of certain essential genes.

\section{( 2 ). The consequence of mitigation of the functional contradiction}

In the wake of the gradual mitigation of the intensified functional contradiction in evolution, the probability of preservation of favourable mutations gradually, and in- 
Evolutionary conservative phenomena in eukaryotic cells

evitably decrease to a level equal to the preservation probability of a neutral mutation, and the mutation is liable to suffer the same fate of random genetic drift. When the functional contradiction has been highly mitigated, evolutionary conservation emerges and the architecture or mechanism will not change unless the functional contradiction is re - intensified. The architecture or mechanism can evolve only when a series (not one) of functionally interrelated favourable mutations are well preserved.

\section{The reduction of evolutionary competence and the genesis of evolutionary conserva- tion}

\section{( 1 ). The limits of evolutionary competence}

Seemingly, there are quite a number of different ways to mitigate an intesified functional condradiction. But in fact, for a particular organism in a particular situation, the available ways are indeed very few. The practical evolutionary way is largely predetermined. For example, in primitive eukaryotes devoid of mitochondria, there were seemingly plenty of possible ways to improve the existing energy - supplying system. One way was to improve various enzymes involved, but in reality, the improvement along this way was very limited. Another way was to create a new kind of organelle to carry out the oxidative phosphorylation. But actually there was no ground for enabling such kind of organelle to emerge, because for the genesis of a special organelle to conduct oxidative phosphorylation, the cell had to be able to utilize oxygen and to carry on this activity. The third way was to live as a parasite in order to get enough energy supply,just as various species of Giardia do today. But in that evolutionary stage of life, the suitable multicellular or unicellular host had not itself emerged yet. It seems likely that in that stage the only permitted way was at first to capture a certain species of aerobic bacterium which had already been able to conduct oxidative phosphorylation, as endosymbiotic energy supplyers, and then to be transformed into mitochondria.

\section{( 2 ) The reduction of evolutionary competence}

The examples described above showed that in a particular situation the permitted evolution is constrained within limits. This permissible scope is chiefly determined by the existing architecture or mechanism. When the architecture or mechanism evolved, the permitted scope would also change. But in no circumstances, the evolutionary competence would be unlimited. Beneficial mutations can be selectively preserved only in the condition that they do not go beyond the scope permitted. Beyond this limited scope they can no longer be preserved discriminately just as neutral mutations in radom genetic drift.

When a certain organic structure becomes highly specialized in evolution, its evolutionary competence becomes quite limited or even reduced to zero. In the later case, the architecture of this organic structure can modulate no more.

\section{Selective units and the genesis of evolutionary conservative phenomena at cellular and subcellular levels in multicellular eukaryotes}

The nature of evolution at the cellular and subcellular levels changed in pace with 
the evolution of multicellularity, since multicellular individuals replaced individual cells as selective units in natural selection. Unless a mutation at the cellular or subcellular level can effect selective advantages to the individual, the preservation of the mutation will not be more beneficial than that of a neutral mutation.

Multicellular eukaryotes are composed of a series of hierachical levels: system, organ, tissue, cell, organelle, complex of macromolecules, and macromolecule. There exist highly sophisticated interactions among these levels. Whether an advantageous mutation can really yield selective advantage is primarily determined by these particular interactions within the particular organism. It seems that, in general, the more intermediate levels exist between the individual and the level which the mutation affects, the less effective in natural selection the mutation would be. A mutation which is highly beneficial to a certain organelle may bestow a greater selective advantage on the unicellular eukaryote, but has rather small or even no influence on the natural selection of multicellular eukaryotes, because there are many intermediate levels between the organelle and the individual. By the way, many mutations in multicellular eukaryotes are neutral mutation just because of that, although they are advantageous or even harmful to the function of certain macromolecules, but have no effect, if any, on the natural selection of the individual.

In unicellualr eukaryotes, the selective unit is the individual cell, and the focal point of evolution is at the cellular or subcellular level. During the period of genesis of eukaryotic cell, the focal points of evolution were on the emergence of the organetles of eukaryotic cells. After this period the focal points were transferred to the cellular divergence and the emergence of various specialized organelles in different groups of protists, e. g. the oral structures in ciliate.

At a stage multicellular eukaryotes had emerged, the focal points of evolution, in most cases, were no longer located at the cellular or subcellular levels. Therefore, the evolutionary conservative phenomena at these levels can easily be in comprehensible in multicellular organism.

To couclude, evolutionary conservative phenomena are frequently observed at cellular and subcellular levels, but this does not diminish the importance of evolutionary viewpoint in studying the biology of eukaryotic cells.

Biological structures, from biomacromolecules to the integrated organisms and their activities, in addition to having adaptive features, have also features not suited to their respective functions. These two aspects bring about the functional contradiction which promote the evolution of these structures and living processes.

The evolutionary conservative phenomena in eukaryotic cells were engendered when: 1) the functional contradiction of the organic structure or process had been considerably mitigated, 2) the evolutionary exploitation of the structure or process was greatly reduced, 3) the individual organisms were the only selective units in natural selection. 
Evolutionary conservative phenomena in eukaryotic cells

\section{ACKNOWLEDGEMENTS}

I would like to express my deep gratitute to Dr David J. Patterson (Dept. of Zoology, University of Bristol, UK) for his most valuable suggestions and discussions.

\section{REFERENCES}

[ 1 ] Kubai DF, Ris H. Division in the dinoflagellate Gyrodinium cohnii. J Cell Biol 1969; 40:508-28.

[2] Oakley BR, Dodge JD. Kinetochores associated with the nuclear envelope in the mitosis of a dinoflagellate. J Cell Biol 1974;63:322-5.

[ 3 ] Gao XP, Li JY. The nuclear division of the marine dinoflagellate Oxyrrhis marina. J Cell Sci 1986; 86:16175 .

[ 4 ] Li JY. The proposal of evolutionary cell biology and its role. Zoological Research 1989; 10: 319-26.

[5] Li JY. Evolutionary cell biology and the "un- evolution phenomena" of organelles in multicellular eukaryotes. In:First APOCB (Asia -Pacific Organization for Cell Biology) Congress (Nov. 3-7, 1990, Shanghai, China) Abstracts. 1990:184.

Received 12-8-1991. Revised 21-12-1991. Accepted 15-1-1992. 